\title{
ASSOCIAÇÃO ENTRE MAUS-TRATOS NA INFÂNCIA E DECLÍNIO COGNITIVO NO IDOSO: UMA REVISÃO INTEGRATIVA
}

Francisco Pascoal Junior

Médico Psiquiatra, mestre e doutorando em Gerontologia Biomédica pela Pontifícia Universidade Católica do Rio Grande do Sul (PUCRS), Brasil.

Guilherme Marcos Nogueira

Educador físico, mestre e doutorando em Gerontologia Biomédica pela Pontifícia Universidade Católica do Rio Grande do Sul (PUCRS), Brasil.

Bruno Luiz Guidolin

Médico psiquiatra, mestre em Gerontologia Biomédica pela Pontifícia Universidade Católica do Rio Grande do Sul (PUCRS), Brasil.

\section{Alfredo Cataldo Neto}

Médico psiquiatra, mestre doutor em Clínica Médica pela Pontifícia Universidade Católica do Rio Grande do Sul (PUCRS), Docente Titular da Escola de Medicina da PUCRS e Coordenador do Grupo de Pesquisa Envelhecimento e Saúde Mental (GPESM) da PUCRS, Brasil.
Autor correspondente

Francisco Pascoal Jr

pascoaljunior@uol.com.br
RESUMO: A violência contra a criança representa um importante problema de saúde pública, assim como o declínio cognitivo. A ligação entre essas variáveis vem sendo analisada cada vez mais, porque o estresse experimentado nos primeiros anos de vida pode estar relacionado a mudanças estruturais, funcionais e epigenéticas nas regiões cerebrais envolvidas na cognição. O objetivo deste estudo foi analisar essa associação, por meio de uma revisão integrativa da literatura, pelo meio da pesquisa bibliográfica das seguintes bases de dados: SciELO, LILACS, PUBMED e PsycINFO. O período de seleção dos artigos compreendeu os últimos cinco anos. No total, foram 33 artigos, dos quais dez eram artigos originais que focaram na relação entre maus-tratos na infância e declínio cognitivo em idosos. A presença de maus-tratos na infância possui associação com o declínio da função cognitiva no idoso, agravando-o sobremaneira e inclusive podendo colocar os indivíduos em risco de declínio cognitivo acentuado. Uma das limitações deste estudo foi a percepção de que a temática ainda carece de mais e melhores estudos que abordem esta relação na prática, por meio de estudos longitudinais.

PALAVRAS-CHAVE: Declínio cognitivo; Idosos; Maus-tratos na infância.

\section{ASSOCIATION BET WEEN MALTREATMENT IN CHILDHOOD AND COGNITIVE DECLINE IN THE ELDERLY: AN INTEGRATIVE REVIEW}

\begin{abstract}
Violence against children and cognitive decline are highly relevant issues for health. The bond between the variables is increasingly being analyzed, since stress early in life may be related to structural, functional and epigenic changes in brain regions involving cognition. Current study analyzes such association through an integrative review of the literature and by bibliographical research retrieved from SciELO, LILACS, PUBMED and PsycINFO during the last five years. Thirty-three original scientific articles were analyzed, with ten related to maltreatment in childhood and cognitive decline in the elderly. Maltreatment in childhood is associated with possible high cognitive decline in old age. A limit in current study was the perception that the theme required more investigation within longitudinal studies.
\end{abstract}

KEY WORDS: Cognitive decline; Elderly; Child Maltreatment.

\section{INTRODUÇÃO}

A violência contra a criança atualmente representa um importante problema de saúde pública, trazendo fortes impactos psicossociais, podendo ser considerada como uso da força física ou do poder, real ou em ameaça, contra si próprio, contra outra pessoa, 
ou contra um grupo ou uma comunidade, que resulte ou tenha qualquer possibilidade de resultar em lesão, morte, dano psicológico, deficiência de desenvolvimento ou privação. Mais do que qualquer outro tipo de violência, a cometida contra a criança, altamente prevalente e uma das condições mais prejudiciais que ela pode experimentar, não se justifica, pois as condições peculiares de desenvolvimento desses cidadãos os colocam em extrema dependência de pais, familiares, cuidadores, do poder público e da sociedade. ${ }^{1,2}$

Os maus-tratos durante a infância - abuso físico e sexual, negligência emocional, perda parental, entre outros - estão associados a fatores responsáveis pelo desenvolvimento de uma série de distúrbios no idoso, como é o caso do declínio cognitivo. Apesar de sua importância, estimar a prevalência de maus-tratos na infância é um desafio pela ausência de um "padrão ouro" claro sobre o que constitui maus-tratos. ${ }^{3}$

No Brasil, as situações de maus-tratos infantis ganharam visibilidade no final da década de 1980, momento em que a Constituição Federal de 1988 e o Estatuto da Criança e do Adolescente (ECA) de 1990 passaram a exigir a notificação de casos suspeitos ou confirmados de violência infantil. A partir de então, as políticas de proteção à criança e ao adolescente começam a se firmar. Um dado importante é que, apesar de manter os altos índices de violência sexual infantil, o Brasil é tido, no âmbito internacional, como um dos países que mais luta contra essa problemática, graças à criação de uma legislação de caráter protetivo. ${ }^{4}$

Em nível mundial, o estudo de Woody et al (2018) apontou que o abuso sexual é a forma mais comumente estudada de maus-tratos, com prevalência de $20,4 \%$ $(13,2 \%$ a $33,6 \%)$ e $28,8 \%(17,0 \%$ a $40,2 \%)$ em meninas norte-americanas e australianas, respectivamente; no entanto, com taxas mais baixas geralmente para meninos. As taxas de abuso físico foram mais semelhantes entre os sexos, exceto na Europa, que foram de 12,0\% (6,9\% a $23,0 \%$ e e $27,0 \%$ ( $7,0 \%$ a $43,0 \%)$ para meninas e meninos, respectivamente, e muitas vezes muito altas em alguns continentes como, por exemplo, 50,8\% (36,0\% a 73,8\%) e $60,2 \%(43,0 \%$ a $84,9 \%)$ para meninas e meninos, respectivamente, na África. Já no Brasil, os dados do Sistema de Informação de Agravos de Notificação (SINAN) apontaram que, somente no ano de 2011, o país registrou 39.281 atendimentos na faixa de 1 a 19 anos idade, que representam $40 \%$ do total de 98.115 atendimentos computados pelo sistema nesse ano., ${ }^{1,3}$

A prevalência de declínio cognitivo na população mundial representa um verdadeiro problema de saúde pública, com uma incidência crescente a cada ano. Um dos fatores que também está relacionado ao aumento dessa prevalência é a constatação do envelhecimento populacional crescente, não somente no Brasil, mas em todo o mundo. Basicamente, o declínio cognitivo é caracterizado pela presença de déficit progressivo nas funções cognitivas (linguagem, motricidade, habilidades visuo-espaciais, dentre outras), com maior ênfase na perda de memória e interferência nas atividades sociais e ocupacionais. ${ }^{5}$

A ligação entre maus-tratos na infância e declínio cognitivo vem sendo analisada, uma vez que o estresse experimentado nos primeiros anos de vida pode estar relacionado a mudanças estruturais, funcionais $\mathrm{e}$ epigenéticas nas regiões cerebrais envolvidas na cognição, conforme visto por Bianco e Canto-de-Souza (2018). ${ }^{6} \mathrm{Em}$ relação a vivências de estresse pós-traumático sofridas, sugere-se uma redução do volume do hipocampo e córtex pré-frontal, uma responsividade exagerada da amídala, com deficiência da sua modulação pelo córtex pré-frontal ventro-medial e hipocampo, hiperfunção do eixo simpato-adrenal e a hipofunção do eixo hi-potálamopituitária-adrenal. Assim, é um transtorno relacionado à ansiedade e memória, que pode correlacionar-se com a velhice e o declínio congntivo nessa fase. ${ }^{6}$

Este tipo de violência resulta em danos na capacidade funcional, tentativas de suicídio, violação de direitos humanos, diminuição da qualidade de vida e elevadas taxas de mortalidade. ${ }^{7}$ Alterações, ao longo do ciclo vital, que podem ter um impacto sinérgico entre si, somado à condição de declínio cognitivo normal no idoso, podem aumentar esse processo e torná-lo patológico.

Os maus-tratos na infância estão associados a traumas para a vida toda de uma pessoa, resultando em mudanças de formas diversas, atingindo todo o ciclo vital, sendo, portanto, de grande relevância tornar conhecidos os impactos e consequências que estes podem trazer na vida do ser humano nas idades mais avançadas. Da mesma 
forma, levanta-se a hipótese de que os maus-tratos na infância trazem consigo uma série de consequências negativas e declínio cognitivo no idoso.

Sabe-se que, via de regra, o comprometimento cognitivo tem o poder de afetar a capacidade funcional do indivíduo no seu dia a dia, implicando perda de independência e autonomia, a qual varia de acordo com o grau de gravidade, com consequente perda da qualidade de vida do idoso. A noção de autonomia, no que tange à interdependência desta com uma memória íntegra, reside na capacidade individual de cuidar de si mesmo, executar tarefas que lhe permitam a adaptação psicosocial e ser responsável pelos próprios atos. ${ }^{8}$

Mas de fato existe relação entre maus-tratos ocorridos na infância e declínio cognitivo no idoso? Estes maus-tratos têm relação direta ou indireta no declínio cognitivo no idoso?

Diante de tal contextualização, o objetivo do presente estudo volta-se para a análise da existência de uma relação entre os maus-tratos ocorridos na infância e o surgimento do declínio cognitivo no idoso.

\section{METODOLOGIA}

Trata-se de uma revisão integrativa. A metodologia adotada para o desenvolvimento do tema será a revisão sistemática da literatura, por meio da adoção da pesquisa bibliográfica nas seguintes bases de dados: SciELO, LILACS, PubMed e PsycINFO. Para a pesquisa nas bases de dados acima relacionadas, foram utilizadas como descritores as seguintes palavras-chave: idosos; declínio cognitivo;cognição;envelhecimento;ma us-tratos na infância.

Como critérios de inclusão, foram utilizados somente artigos publicados na língua portuguesa e língua inglesa. A linguagem dos artigos configurou-se, também, como um critério de seleção adotado.O período de seleção dos artigos compreendeu os últimos cinco anos, ou seja, a partir de 2013 até 2018. A opção por este período de cinco anos das publicações deveu-se à necessidade de se encontrar fontes e material recente, atualizado, envolvendo pesquisas que abordem esta temática.

Resumos e textos que se demonstraram completos foram incluídos em uma nova seleção composta por quatro critérios de inclusão/exclusão. $\mathrm{Na}$ presente etapa excluiu-se: (1) estudos em que não era possível a recuperação do texto completo; (2) revisões da literatura que não tratem sobre seres humanos; (3) estudos em que o objetivo principal fora a avaliação de outros vocábulos do que os não utilizados nas palavraschave; (4) estudos que não apresentem relação entre maus-tratos e declínio cognitivo.

\section{RESULTADO}

No total, foram 33 artigos (Figura 1), dos quais exclusivamente dez eram artigos originais que focaram na relação entre maus-tratos na infância e declínio cognitivo em idosos. Os detalhes clínicos e experimentais desses dez artigos originais estão descritos no Quadro 1.

Pelo cruzamento dos descritores "idosos", "cognição" e "maus-tratos na infância", foi possível obter uma amostra de 31 artigos. Já no cruzamento dos descritores "envelhecimento", "declínio cognitivo" e "maus-tratos na infância", a amostra obtida foi de dois artigos. Para cruzamento dos descritores, não foram utilizados operadores booleanos.

Dentre os 33 estudos, 15 foram selecionados para uma avaliação mais profunda, utilizando-se os critérios de seleção mencionados anteriormente. Ressaltase ainda que os 18 artigos que foram excluídos desta amostra não atenderam aos critérios de elegibilidade adotados, bem como não apresentavam sua temática principal compatível com os objetivos buscados para este levantamento, ou seja, não tinham relação direta com a temática da abordagem dos maus tratos na infância e seus impactos na vida senil.

Em seguida, realizou-se a exclusão de cinco estudos que se apresentaram duplicados entre as bases. Os resumos e textos destes estudos restantes foram analisados levando-se em consideração os critérios de inclusão/exclusão.

Após a realização da seleção, restaram dez estudos, sendo que estes foram realmente utilizados, por se enquadrarem dentro dos critérios de exclusão/ inclusão.

Durante todo o processo, dois autores revisaram a busca e a análise dos artigos de forma independente. 
A Figura 1, abaixo, apresenta uma representação esquemática dos artigos encontrados, incluidos e excluídos da amostra final.

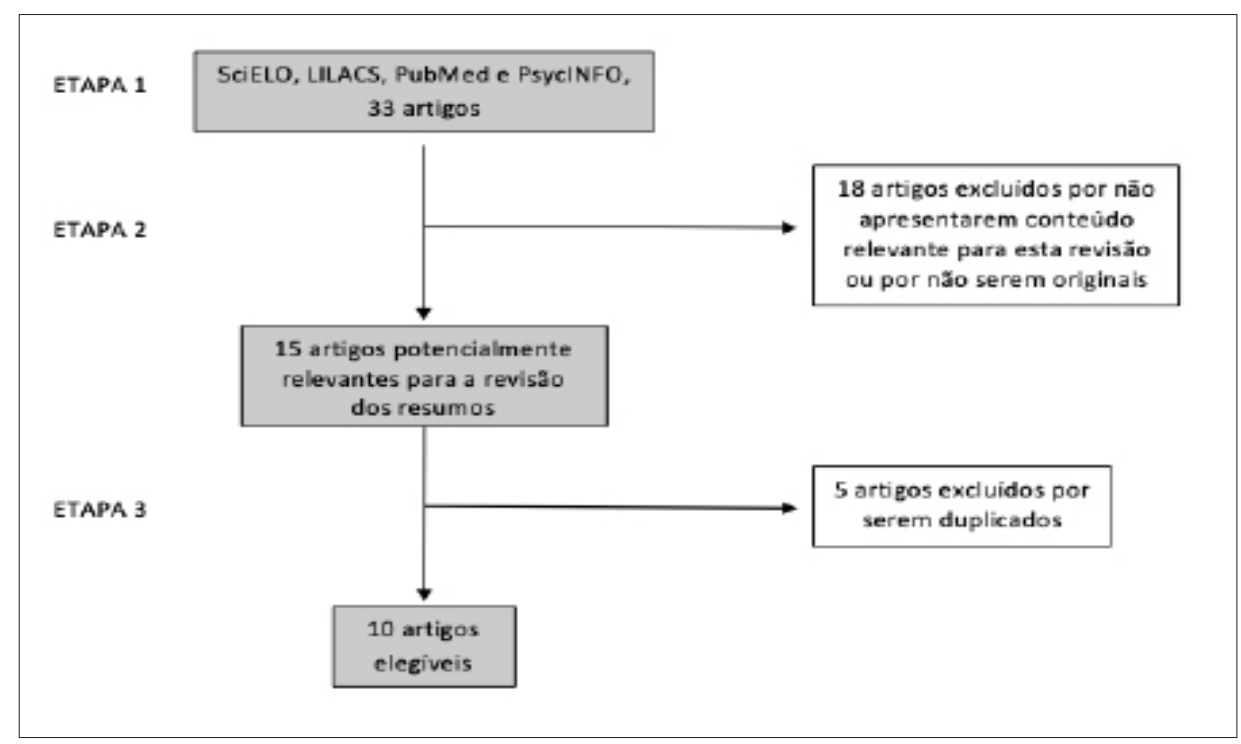

Figura 1. Fluxograma com trajeto da pesquisa bibliográfica e critérios de inclisão e exclusão dos artigos encontrados.

Apesar de poucos artigos que abordam o assunto sobre a presença de maus-tratos na infância e sua relação com o desenvolvimento do declínio cognitivo em idosos, foi possível observar, nos artigos encontrados e estudados, uma forte associação nesse sentido. Essa relação envolve não só o declínio cognitivo, mas também o desenvolvimento de outros transtornos psiquiátricos, como os depressivos e os associados ao estresse, que acabam por atuarem em sinergismo com o impacto deletério dos efeitos dos maus-tratos na infância ao longo de todo o ciclo vital.

Nesse aspecto, o Quadro 1, a seguir, mostra os estudos e respectivos resultados encontrados, relacionados à temática maus-tratos na infância e declínio cognitivo no idoso.

Quadro 1. Características dos artigos originais, selecionados na pesquisa bibliográfica, que abordam o tema maus-tratos na infância e sua associação com o declínio cognitivo em idosos, entre os anos de 2013 a 2018

\begin{tabular}{|c|c|c|c|}
\hline Título & Referência (ano) & Objetivo geral & Resultados \\
\hline $\begin{array}{l}\text { Childhood Trauma is Asso- } \\
\text { ciated With Poorer Cognitive } \\
\text { Performance in Older Adults }\end{array}$ & $\begin{array}{l}\text { Petkus Aj, Lenze EJ, Buthers } \\
\text { MA, Twanley EW, Wetherell JL } \\
(2018)^{22}\end{array}$ & $\begin{array}{l}\text { Investigar o trauma infantil em } \\
\text { relação ao desempenho cognitivo } \\
\text { em idosos. }\end{array}$ & $\begin{array}{l}\text { Impacto deletério do trauma infan- } \\
\text { til na saúde cerebral na velhice }\end{array}$ \\
\hline $\begin{array}{l}\text { The influence of childhood } \\
\text { abuse on cortisol levels and the } \\
\text { cortisol awakening response in } \\
\text { depressed and nondepressed } \\
\text { older adults }\end{array}$ & $\begin{array}{l}\text { Wiellard I, Schaakxs R, Comi- } \\
\text { js HC, Stek ML, Rhebergen D. } \\
(2017)^{16}\end{array}$ & $\begin{array}{l}\text { Examinar o impacto do abuso } \\
\text { infantil e sua interação com a } \\
\text { depressão nos níveis de cortisol } \\
\text { em idosos }\end{array}$ & $\begin{array}{l}\text { Idosos com histórico de abuso na } \\
\text { infância podem ser mais afetados } \\
\text { negativamente por eventos estres- } \\
\text { santes, o que se reflete na desregu- } \\
\text { lação do eixo HPA }\end{array}$ \\
\hline $\begin{array}{l}\text { Relação entre violência e fun- } \\
\text { ção cognitiva em idosos }\end{array}$ & $\begin{array}{l}\text { Faustino AM, Moura LBA, } \\
\text { Gandolfi L. }(2016)^{20}\end{array}$ & $\begin{array}{l}\text { Determinar se existe relação } \\
\text { entre cognição em idosos e expo- } \\
\text { sição à violência }\end{array}$ & $\begin{array}{l}\text { Houve associação significativa } \\
\text { entre maus-tratos e mudança na } \\
\text { função cognitiva de idosos }\end{array}$ \\
\hline $\begin{array}{l}\text { Narrative of self-neglect: pa- } \\
\text { tterns of traumatic personal } \\
\text { experiences and maladaptive } \\
\text { behaviors in cognitively intact } \\
\text { older adults }\end{array}$ & $\begin{array}{l}\text { Lien C, Rosen T, Bloemen } \\
\text { EM, Abrams RC, Pavlou M, } \\
\text { Lachs MS (2016) })^{19}\end{array}$ & $\begin{array}{l}\text { Identificar padrões de auto-ne- } \\
\text { gligência, através de narrativas } \\
\text { de idosos }\end{array}$ & $\begin{array}{l}\text { Foram identificados padrões de } \\
\text { experiências pessoais traumáticas } \\
\text { e comportamentos desadaptativos } \\
\text { em idosos anto-negligentes }\end{array}$ \\
\hline $\begin{array}{l}\text { How do negative emotion rela- } \\
\text { te to dysfunctional posttrauma } \\
\text { cognitions? An examination of } \\
\text { interpersonal trauma survivors }\end{array}$ & $\begin{array}{l}\text { Beck JG, Reich CM, Woo- } \\
\text { dward MJ, Olsen AS, Jones } \\
\text { JM, Patton SC }(2015)^{31}\end{array}$ & $\begin{array}{l}\text { Examinar associações entre } \\
\text { emoções negativas e cognições } \\
\text { disfuncionais relacionadas ao } \\
\text { trauma }\end{array}$ & $\begin{array}{l}\text { Níveis mais altos de emoções nega- } \\
\text { tivas foram significativamente asso- } \\
\text { ciados a disfunções cognitivas }\end{array}$ \\
\hline
\end{tabular}


(Conclusão)

\begin{tabular}{|c|c|c|c|}
\hline Título & Referência (ano) & Objetivo geral & Resultados \\
\hline $\begin{array}{l}\text { Childhood abuse in late-life } \\
\text { depression }\end{array}$ & $\begin{array}{l}\text { Comijsa HC, Exel E, Van der } \\
\text { Mast RC, Paauw MA, Voshaar } \\
\text { O, Stek ML }(2013)^{18}\end{array}$ & $\begin{array}{l}\text { Estudar a relação entre abuso na } \\
\text { infância e depressão tardia }\end{array}$ & $\begin{array}{l}\text { Houve forte relação entre depres- } \\
\text { são em idosos e abuso na infância, } \\
\text { com associação a um aumento } \\
\text { no número de doenças crônicas } \\
\text { comórbidas }\end{array}$ \\
\hline $\begin{array}{l}\text { Effect of maltreatment on brain } \\
\text { structure in adult patients with } \\
\text { major depressive disorder and } \\
\text { healthy participants }\end{array}$ & $\begin{array}{l}\text { Chaney A, Carballedo A, Ami- } \\
\text { co F, Fagan A, Skokauskas N, } \\
\text { Meaney J, Frodl T }(2014)^{17}\end{array}$ & $\begin{array}{l}\text { Investigar a relação entre de- } \\
\text { pressão e história de maus-tratos } \\
\text { na infância e suas mudanças } \\
\text { estruturais }\end{array}$ & $\begin{array}{l}\text { Há alteração estrutural no encéfalo } \\
\text { em indivíduos que sofrem maus- } \\
\text {-tratos na infância em comparação } \\
\text { com aqueles que não sofreram }\end{array}$ \\
\hline $\begin{array}{l}\text { The impact of the developmen- } \\
\text { tal timing of trauma exposure } \\
\text { on PTSD symptoms and psy- } \\
\text { chosocial functioning among } \\
\text { older adults }\end{array}$ & $\begin{array}{l}\text { Ogle CM, Rubin DC, Siegler } \\
\text { IC }(2013)^{15}\end{array}$ & $\begin{array}{l}\text { Examinar a associação entre } \\
\text { transtorno do estresse pós-trau- } \\
\text { mático (TEPT) e funcionamento } \\
\text { psicossocial em idosos }\end{array}$ & $\begin{array}{l}\text { Idosos que sofreram evento trau- } \\
\text { mático mais angustiante durante } \\
\text { a infância exibiram sintomas mais } \\
\text { severos de transtornos de estresse } \\
\text { pós traumático }\end{array}$ \\
\hline $\begin{array}{l}\text { Cognitive function is preserved } \\
\text { in older adults with a reported } \\
\text { history of childhood sexual } \\
\text { abuse }\end{array}$ & $\begin{array}{l}\text { Feeney J, Kamiya Y, Robert- } \\
\text { son IH, Kenny RA }(2013)^{12}\end{array}$ & $\begin{array}{l}\text { Investigar a função cognitiva em } \\
\text { idosos com história de abuso } \\
\text { sexual na infância }\end{array}$ & $\begin{array}{l}\text { A ocorrência de abuso sexual na } \\
\text { infância foi associada com a pior } \\
\text { saúde psicológica nos idosos }\end{array}$ \\
\hline $\begin{array}{l}\text { Maus-tratos na infância e fun- } \\
\text { cionamento cognitivo tardio: } \\
\text { uma revisão sistemática }\end{array}$ & $\begin{array}{l}\text { Irigaray TQ, Pacheco JB, } \\
\text { Grassi-Oliveira R, Fonseca } \\
\text { RP, Leite JCC, Kristensen CH } \\
(2013)^{25}\end{array}$ & $\begin{array}{l}\text { Investigar a relação entre maus- } \\
\text {-tratos e funcionamento cogniti- } \\
\text { vo através de revisão sistemática } \\
\text { da literatura }\end{array}$ & $\begin{array}{l}\text { Idosos que sofreram maus-tratos } \\
\text { na infância demonstraram um } \\
\text { perfil cognitivo inferior }\end{array}$ \\
\hline
\end{tabular}

\section{DISCUSSÃO}

A cognição acaba por envolver o funcionamento mental, como, por exemplo, as habilidades de pensar, lembrar, raciocinar, lembrar, perceber, e, responder a qualquer tipo de estímulos externos. Embora possa ocorrer mudanças no desempenho cognitivo em razão do envelhecimento, tal prejuízo não afeta realmente a vida cotidiana dos idosos e de seus familiares. ${ }^{6}$

Para a maioria dos idosos, as capacidades cognitivas não são afetadas, mesmo tendo maior dificuldade em armazenar e recuperar as informações. Existe uma grande variabilidade em relação ao funcionamento cognitivo na terceira idade, sendo que o declínio poderá ocorrer em diferentes áreas. Em estudo de Rosa et al (2016), verificouse maior prevalência de transtornos mentais e de realização de tratamento psiquiátrico nos idosos com prejuízos

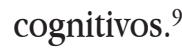

Neste sentido, ao se levar em consideração a influência dos maus-tratos durante a infância e o declínio cognitivo durante a fase idosa, há uma relação entre entre esses domínios. A negligência emocional foi a forma de maus-tratos que mais influenciou a presença de traços de psicopatia e que mais se associou a prejuízos de habilidades sociais específicas. O volume de substância branca de regiões do hemisfério esquerdo (giro angular, precuneus e lobo parietal inferior) correlacionou-se negativamente com a afetividade, de acordo com estudo realizado por Scarparo(2016).$^{10}$

Dentro deste contexto, o efeito em longo-prazo da exposição à violência parental e abuso físico parental na infância é o risco elevado para perpetração da violência na idade adulta, seja em seus relacionamentos íntimos, seja com a perpetração contra os próprios filhos. ${ }^{11}$

Pela amostra selecionada, foi possível observar que, embora escassos, há estudos que abalizam a tese de que os impactos dos maus-tratos na infância estão associados ao comprometimento não somente do fator cognitivo, mas também a uma série de impactos negativos que se perpetuarão por toda a vida do indivíduo.

O envelhecimento natural acaba por trazer impactos nos mais variados órgãos e sistemas do corpo humano. No desempenho cognitivo não é diferente, já que o processo de envelhecimento irá também trazer uma série de 
limitações para os idosos, de uma forma geral.

No entanto, o envelhecimento e seu decréscimo cognitivo não são capazes de afetar realmente a vida cotidiana dos idosos e de seus familiares. Para a maioria dos idosos, as capacidades cognitivas não são afetadas, mesmo tendo maior dificuldade em armazenar e recuperar as informações. Todavia, quando há um comprometimento cognitivo em decorrência de um ou mais fatores deletérios, observa-se um quadro diferente.

Estes fatores deletérios poderiam estar associados aos maus-tratos que os idosos podem ter passado durante sua infância, havendo maior propensão para apresentarem piores quadros de saúde psíquica em idosos que sofreram abuso sexual na infância, em comparação com aqueles que não relataram história de abuso sexual infantil. ${ }^{12}$

Háoconsenso de queo eixohipotálamo-pituitáriaadrenal (HPA) desempenha um papel fundamental na resposta aos estímulos psíquicos externos e internos, podendo ter papel importante no desencadeamento de episódios psiquiátricos em indivíduos predispostos. ${ }^{13}$

A vivência em situações de estresse, especialmente nas fases mais precoces do desenvolvimento humano, pode também ter relação direta com a ocorrência de alterações no eixo HPA, que serão persistentes por toda a vida do indivíduo. Estas alterações podem causar diminuição da capacidade do indivíduo de participar de forma responsiva e positiva frente às situações de estresse durante todo o percurso de sua vida. ${ }^{14}$

Todas estas questões chamam a atenção para uma possível associação entre a ocorrência de traumas, agressões, estresse excessivo, enfim, maus-tratos vivenciados na infância e a possível relação destes fatores com uma possível alteração no eixo HPA e o desencadeamento consequente de demências já na vida adulta e senil.

Nesse aspecto, idosos que experimentaram seu evento traumático mais angustiante durante a infância exibiram sintomas mais severos de Transtorno do Estresse Pós-Traumático (TEPT), que possui relação com a perda cognitiva hipocampal, pelo efeito inflamatório deletério e neurotóxico cortisol advindo do eixo HPA, e menor felicidade subjetiva em comparação com adultos mais velhos que sofreram o trauma mais angustiante após a transição para a vida adulta. ${ }^{15}$

Nesse aspecto, o abuso na infância estaria associado a níveis basais de cortisol mais baixos ao despertar e adultos mais velhos, com histórico de abuso na infância, poderiam ser mais afetados negativamente pelo estresse ou por eventos estressantes, o que se refleteria na desregulação do eixo HPA. ${ }^{16}$

Da mesma forma, foi encontrado significativamente diminuído o volume de substância cinzenta no hipocampo e aumentado de forma importante no córtex pré-frontal dorsomedial (DMPFC) e no córtex orbitofrontal (OFC) em participantes que sofreram maustratos na infância em comparação com aqueles que não tiveram, o que sugere alterações estruturais no cérebro de idosos associadas a maus-tratos sofridos na infância. ${ }^{17}$

Foi observado ainda uma relação positiva entre o abuso na infância e a depressão de início tardio, muito associada à perda cognitiva importante, a sintomas de anedonia e apatia, além de comorbidades, o que poderia sugerir o bem-estar psicológico poder ser mantido por toda a meia-idade, mas também pode ser perturbado mais tarde na vida, mesmo tendo o evento traumático ocorrido na infância. ${ }^{18} \mathrm{Ao}$ se levar em consideração a influência dos maus-tratos durante a infância e o declínio cognitivo durante a fase idosa, há uma relação positiva, mas as pessoas podem permanecer estáveis ao longo da vida adulta. ${ }^{10}$

Em suma, o desgaste cognitivo, particularmente, pode levar o indivíduo a cometer erros básicos de processamento de informações, fruto de uma assimilação mais lenta dos aspectos relevantes da tarefa, ou até mesmo pela impossibilidade de detecção de detalhes imprescindíveis ao planejamento de uma determinada estratégia. Estes fatos, em conjunto, podem contribuir para que o indivíduo decida sem que tenha compreendido por completo a conjuntura em que problema está envolvido.

Foram identificados padrões de experiências pessoais traumáticas e comportamentos desadaptativos, relacionados à autonegligência. ${ }^{19}$ Dessa forma, experiências, percepções e comportamentos desenvolvidos ao longo da vida podem contribuir para a autonegligência dos idosos. Nesse contexto, foi evidenciada a associação entre a violência sexual, negligência e autonegligência e mudanças na função cognitiva de idosos. ${ }^{20} \mathrm{Da}$ mesma forma, também foi visto que o estresse, em razão de maus tratos durante a infância, tende a acelerar os processos básicos do envelhecimento, principalmente em termos cognitivos, resultando na 
vulnerabilidade do idoso. ${ }^{21}$

O trauma infantil foi significativamente associado a pior desempenho nas medidas de velocidade de processamento, atenção e funcionamento executivo em idosos. Eventos traumáticos relatados na infância estariam, portanto, associados a pior desempenho cognitivo em idosos. Esses resultados demonstram o impacto deletério do trauma infantil na saúde mental na velhice. ${ }^{22}$

Desta forma, o declínio cognitivo traz consigo o enfrentamento entre o envelhecimento saudável e patológico, abrangendo o surgimento de uma demência leve, como observam Ribeiro et al (2016), afirmando que os idosos vivenciam o envelhecimento e a finitude com estratégias de enfrentamento que podem gerar tanto resultados desfavoráveis quanto favoráveis à saúde, como perda financeira; redução da cognição; perda da sensação de domínio; perda da sensação de ser útil; redução do bem-estar subjetivo e da qualidade de vida. ${ }^{23}$

Na existência de maus-tratos durante a infância, o declínio cognitivo apresentaria maior incidência especialmente na parte da memória, que se associa ao declínio em outras funções cognitivas. Assim, o declínio cognitivo pode comprometer outras funções cognitivas, possibilitando o surgimento de outras alterações, sobretudo as psiquiátricas, que acabam por ser resultado de vivências negativas de maus-tratos durante a vida, conforme evidenciado por Marques (2017). ${ }^{24}$

Essas alterações psiquiátricas como fatores preditivos para o prejuízo cognitivo em idosos, contribuem para piorar as atividades funcionais e a independência destes pacientes, o que, consequentemente, levará à piora dos sintomas iniciais e a um ciclo vicioso que se estende por todo o mote vital. ${ }^{24}$

Os idosos sujeitos a maus-tratos durante a infância apresentam queixa de memória, principalmente comprometimento da memória em testes, em razão da função cognitiva global preservada. Em tais casos, a perda de memória episódica é considerada como maior que o esperado, mas não preenchendo critérios para o diagnóstico de demência. Da mesma forma, quando há maus-tratos na infância, observa-se decréscimo na capacidade de memória verbal episódica, memória de trabalho e atenção, conforme verificado por Irigaray et al (2013). ${ }^{25}$

As associações clínicas das queixas de problemas com a memória estão relacionadas com a questão dos maus-tratos, atingindo principalmente as dificuldades subjetivas de memória, como afirmam Cunha et al (2015) ${ }^{26}$ Essa dificuldade amnéstica se relaciona a sintomas depressivos e ansiosos, que pioram o desempenho cognitivo em idosos. Além disso, ocorrem déficits em aividades de vida diárias (AVDs) avançadas e instrumentais, comprometendo a qualidade de vida e constituindo-se em risco de estágio pré-demencial. Dessa forma, o idoso que apresenta declínio cognitivo leve, tende, de forma gradativa, a perder a capacidade de realização de suas atividades cotidianas, apresentando seus relacionamentos sociais afetados e podendo se isolar socialmente, atividade que é fator de proteção para a incapacidade funcional e o prejuízo cognitivo em idosos, conforme afirmam Brito et al (2012). ${ }^{27} \mathrm{~A}$ dependência constitui, portanto, fator de risco significativo para a piora do declínio cognitivo na população idosa e relações sociais satisfatórias acabam por promoverem melhores condições de saúde, na perspectiva de influenciarem diretamente na esfera cogitiva. ${ }^{27}$

O risco de desenvolvimento de declínio cognitivo encontra associação a fatores considerados intrínsecos e extrínsecos do ser humano, dentre os quais os maustratos na infância teriam papel significativo, além de baixa escolaridade e hipertensão arterial, dentre outras. Como visto por Nasciemento et al (2015), a predominância maior ocorre em mulheres, estando associada com a incapacidade funcional, presença de comorbidades, falta de contato social, atividade intelectual pobre, entre outros. $^{28}$

Alves et al (2016) evidenciaram a existência de relações entre o estilo de vida durante a infância e a capacidade cognitiva ${ }^{29}$ Crianças provenientes de famílias desfavorecidas têm menos acesso a uma variedade de materiais lúdicos e pedagógicos, desde a infância até a adolescência. Em ambientes familiares e a contextos sociais mais favorecidos, econômica e culturalmente, elas se beneficiam de mais oportunidades de interação com as ferramentas e os artefatos socioculturaus, podendo, dessa forma, otimizar o seu desenvolvimento cognitivo. Assim, determinadas ocorrências negativas durante a infância possibilitariam maior possibilidade de surgimento, durante todo o ciclo vital, incluindo a fase idosa, de um prejuízo cognitivo, mais acentuado na velhice. ${ }^{29}$ Dessa forma, quanto mais saudável for a vida durante a infância, sem maiores ocorrências negativas, melhor será o 
desempenho dos idosos em habilidades cognitivas, como linguagem, memória e atenção.

Nesse mesmo contexto, a privação nutricional precoce na infância, decorrente de negligência, pode contribuir para o comprometimento cognitivo entre idosos. Considerando a nutrição como fator de influência ao desenvolvimento cognitivo infantil, aceita-se a ideia de que a desnutrição está associada à patologia estrutural e funcional do cérebro, resultando em dano tecidual, retardo do crescimento, diferenciação desordenada, redução de sinapses e neurotransmissores sinápticos, mielinização retardada e redução do desenvolvimento geral da arborização dendrítica do cérebro em desenvolvimento. ${ }^{30}$ Desvios nutricionais, portanto, induzem alterações estruturais e funcionais cerebrais, que por sua fez podem prejudicar a memória e, consequentemente, afetar o aprendizado principalmente em fases de desenvolvimento cognitivo, perdurando até a fase idosa e podendo implicar num maior prejuízo cognitivo nessa etapa da vida. ${ }^{30}$

Ao se examinar a questão da influência de maus-tratos no decorrer da infância e o surgimento do declínio cognitivo na fase idosa, Ogle et al (2013) verificam que a probabilidade de existir essa associação aumenta consideravelmente, pois ocorre certo grau de comprometimento no aspecto psíquico do indivíduo. Neste contexto, torna-se compreensível entender que os eventos traumáticos e os maus-tratos na infância causam impactos sobremodo avassaladores em longo prazo. ${ }^{15}$

Essas consequências podem estar associadas, juntamente com o sentimento de culpa, a uma maior probabilidade para a perpetuação tanto da culpabilização como do quadro depressivo e do impacto no desempenho cognitivo, atuando todos esses fatores em uma sinergia negativa, como relaram Beck et al (2015) ${ }^{31}$ Dessa forma, a avaliação dos riscos relacionados aos maus-tratos contra os idosos é imprescindível, pois por meio deles poderá ser prestada uma assistência adequada; essa percepção desde a infância se faz necessária com mais ênfase ainda, com base nas consequentes alterações cognitivas e psicológicas que podem ocorrer no ciclo vital. ${ }^{31}$

Se a saúde de um indivíduo resulta de condições biológicas, socioculturais e psíquicas, é possível, então, levantar a hipótese de que o convívio social e diferentes atividades de lazer, desde a infância, possam contribuir como mais um fator de proteção para o declínio cognitivo no idoso. Principalmente, se considerados os fatores associados a esse declínio, que evidenciam menor escolaridade, menor suporte social, histórico de saúde negativo, menor engajamento social, estilo de vida negativo, pior saúde percebida, mais queixas subjetivas de memória, pior saúde mental e mais sintomas depressivos. Tais fatores, quando ausentes,podem, então, diminuir consideravelmente a possibilidade de surgimento e/ou a piora do declínio cognitivo na fase idosa.

\section{CONCLUSÃO}

Com base nos resultados obtidos, essa revisão contribuiu para o direcionamento que, de fato, os maustratos na infância possuem associação com o declínio da função cognitiva no idoso, agravando-a sobremaneira e inclusive podendo colocar os indivíduos em risco de autonegligência. Esses achados são consistentes com relatos anteriores de alterações neuroanatômicas em indivíduos idosos. Este risco elevado de maior declínio cognitivo parece ser particularmente relevante quando o trauma advém da infância, percorrendo a vida como um todo, até a fase idosa.

Os resultados desse estudo reforçam a ideia de que determinantes sociais da saúde devem ser considerados na análise da relação entre função cognitiva e maus-tratos na infância ao longo do ciclo da vida, sobretudo levando-se em conta a natureza duradoura de eventos traumáticos no início da vida. Nesse aspecto, estão compreendidos os familiares, a população abrangente $\mathrm{e}$ os profissionais de saúde envolvidos no cuidado, além disso, tanto os profissionais que atuam na atenção básica, como na situação de emergência necessitam de capacitação específica para atuar identificando, avaliando, prevenindo e diagnosticando as situações de violência e, assim, informar as autoridades para as possíveis medidas. Como afirmam Grilo et al (2015), é através da notificação que a violência ganha visibilidade e possibilita a criação de políticas públicas voltadas ao assunto. ${ }^{32}$

Portanto, a detecção precoce, pela ação das redes de saúde,é fundamental para prevenir tanto comportamentos de risco que possam sinalizar uma condição de maus-tratos na infância, como o declínio cognitivo no idoso, levando-se em conta essa associação à adversidade traumática precoce. Infelizmente, os maustratos percorrem toda a vida, o tempo não cura; apenas 
esconde.

Como fator limitante deste estudo, destaca-se a percepção de que a temática ainda carece de mais estudos que abordem esta relação temporal, principalmente por meio de estudos longitudinais.

\section{AGRADECIMENTOS}

O presente trabalho foi realizado com apoio da Coordenação de Aperfeiçoamento de Pessoal Nivel Superior - Brasil (Capes).

Instituto de Geriatria e Gerontologia (IGG); Escola de Medicina da Pontifícia Universidade Federal do Rio Grande do Sul (PUCRS).

\section{REFERÊNCIAS}

1. Nunes AJ, Sales MCV. Violência contra crianças no cenário brasileiro Ciência \& Saúde Coletiva. 2016; 21(3):871-880.

2. Dias A, Mooren T, Kleber RJ. Public health actions to mitigate long-term consequences of child maltreatment. J Public Health Policy. 2018; 39(3): 294-303.

3. Moody G, Cannings-John R, Hood K, Kemp A, Robling M. Establishing the international prevalence of self-reported child maltreatment: a systematic review by maltreatment type and gender. BMC Public Health. 2018; 18(1): 1164.

4. Souza GW, Macedo S. Grupo interventivo com genitores (as) de crianças vítimas de violência sexual. Rev. abordagem gestalt. 2018; 24(3):265-274.

5. Radanovic M, Stella F, Forlenza O.

Comprometimento cognitivo leve. Revista De Medicina. 2015; 94(3): 162-168.

6. Bianco MB, Canto-de-Souza ALM. Ansiedade, memória e o transtorno de estresse póstraumático. Revista CES Psicologia. 2018; 11(2): 5365.

7. Castro, VC, Rissardo LK, Carreira L. Violência contra os idosos brasileiros: uma análise das internações hospitalares. Rev. Bras. Enferm. 2018; 71(2): 777-
785.

8. Machado JC, Ribeiro RCL, Cotta RM, Leal PFG. Declínio cognitivo de idosos e sua associação com fatores epidemiológicos em Viçosa, Minas Gerais. Revi Bras. Geriatr. Gerontol. 2011; 14(1): 109-121.

9. Rosa TSM, dos Santos Filha VAV, de Moraes AB. Prevalence and factors associated with cognitive impairments in the elderly of charity asylums: a descriptive study. Ciência \& saúde coletiva. 2018; 23 (11).

10. Scarparo MO. Comportamento social e volume de substância branca cerebral em adolescentes vítimas de maus tratos. São Paulo: University of São Paulo, Faculdade de Medicina. 2016.

11. Waikamp V, Serralta FB. Repercussões do trauma na infância na psicopatologia da vida adulta. Ciencias Psicológicas. 2018; 12(1): 137-144.

12. Feeney J, Kamiya Y, Robertson IH, Kenny RA. Cognitive function is preserved in older adults with a reported history of childhood sexual abuse. J Trauma Stress. 2013; 26(6): 735-43.

13. Tofoli SMC. Depressão, Estresse Precoce, Eixo Hipotálamo-Pituitária-Adrenal e a Resposta Terapêutica: avaliações psicométricas e psiconeuroendócrinas. Dissertação (Mestrado). Ribeirão Preto: Universidade de São Paulo, Faculdade de Medicina. 2012; 180f.

14. Baes CVW. Estresse precoce e alterações do eixo hipotálamo-pituitária-adrenal (HPA) na depressão. Dissertação (Mestrado). Ribeirão Preto: Universidade de São Paulo, Faculdade de Medicina. 2012; 163f.

15. Ogle CM, Rubin DC, Siegler IC. The impact of the developmental timing of trauma exposure on PTSD symptoms and psychosocial functioning among older adults. Developmental Psychology. 2013; 49(11): 2191-2200.

16. Wiellard I, Schaakxs R, Comijs HC, Stek ML, Rhebergen D. The influence of childhood abuse on cortisol levels and the cortisol awakening response in depressed and nondepressed older adults. The 
World Journal of Biological Psychiatry : the Official Journal of the World Federation of Societies of Biological Psychiatry. 2017; 25: 1-10.

17. Chaney A, Carballedo A, Amico F, Fagan A, Skokauskas N, Meaney J, Frodl T. Effect of childhood maltreatment on brain structure in adult patients with major depressive disorder and healthy participants. J Psychiatry Neurosci. 2014; 39(1): 50-9.

18. Comijsa HC, Exel E, Van der Mast RC, Paauw MA, Voshaar O, Stek ML. Childhood abuse in late-life depression. Journal of Affective Disorders. 2013; 147(1-3):241-246.

19. Lien C, Rosen T, Bloemen EM, Abrams RC, Pavlou M, Lachs MS. Narratives of Self-Neglect: Patterns of Traumatic Personal Experiences and Maladaptive Behaviors in Cognitively Intact Older Adults. J Am Geriatr Soc. 2016; 64(11): 195-200.

20. Faustino AM, Moura LBA, Gandolfi L. Relação entre violência e função cognitiva em idosos. Rev. enferm. UFPE on line. 2016; 10(5): 1717-1723.

21. ML Levandowski. Maus-tratos na infância, estresse e envelhecimento celular. Tese (Doutorado) Programa de Pós-Graduação em Psicologia, PUCRS. 2018.

22. Petkus AJ, Lenze EJ, Butters MA, Twamley EW, Wetherell JL. Childhood Trauma Is Associated With Poorer Cognitive Performance in Older Adults. The Journal of Clinical Psychiatry. 2018; 79(1).

23. Ribeiro, MS, Borges, MS, de Araújo TCCF, Souza MCS. Estratégias de enfrentamento de idosos frente ao envelhecimento e à morte: revisão integrativa. Revista Brasileira de Geriatria e Gerontologia, Rio de Janeiro. 2017; 20(6).

24. Marques RC. Repercussões na saúde de experiências traumáticas na infância. Tese (Doutorado) Faculdade de Medicina da Universidade de Coimbra. 2017.

25. Irigaray TQ, Pacheco JB, Grassi-Oliveira R, Fonseca $\mathrm{RP}$, Leite JCC, Kristensen CH. Child maltreatment and later cognitive functioning: a systematic review. Psicologia: Reflexão e Crítica. 2013; 26(2): 376-387.

26. Cunha FCM, Cintra MTG, Dornelas JM, Assis MG, Cassiano JG, Nicolato R, Malloy-Diniz LF, Moraes EN, Bicalho MAC. Declínio funcional em idosos com comprometimento cognitivo leve. Revista Med Minas Gerais. 2015; 25(3).

27. TRP de Brito, SCI Pavarini. Relação entre apoio social e capacidade funcional de idosos com alterações cognitivas. Revista Latino-Americana de Enfermagem. 2012; 20(4).

28. Nascimento RAS, Batista RTS, Rocha SV, Vasconcelos LRC. Prevalência e fatores associados ao declínio cognitivo em idosos com baixa condição econômica: estudo MONIDI. Jornal Brasileiro de Psiquiatria. 2015.

29. Alves AF, Lemos GC, Brito L, Martins AA, Almeida LS. Desempenho Cognitivo na Infância: A Mãe e o Meio Urbano fazem a Diferença. Psicologia: Teoria e Pesquisa. 2016; 32 (3).

30. Ramos ICS.Interação entre o estado nutricional em crianças a partir da segunda infância e alterações de memória. Trabalho de Conclusão de Curso, Núcleo de Nutrição, Universidade Federal de Pernambuco. Vitória de Santo Antão, 2017.

31. Beck JG, Reich CM, Woodward MJ, Olsen AS, Jones JM, Patton SC. How do negative emotions relate to dysfunctional posttrauma cognitions? An examination of interpersonal trauma survivors. Psychol Trauma. 2015; 7(1): 3-10.

32. Grilo PM, Lombardi Filho I. Maus-tratos a idosos: perfil das vítimas, vinculo com o agressor e atuação dos profissionais. Estudos interdisciplinaresdo envelhecimento, Porto Alegre. 2015; 20(2): 611-624.

Recebido em: 21/02/2019

Aceito em: 26/06/2019 